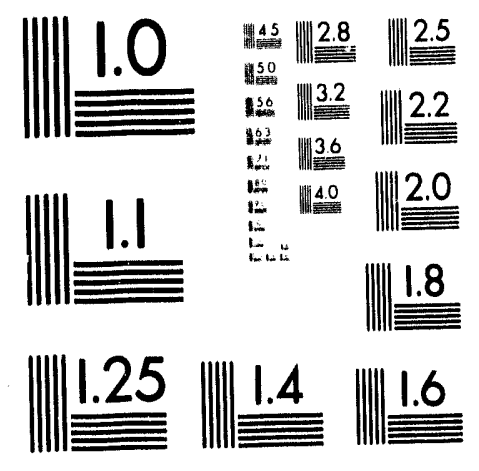



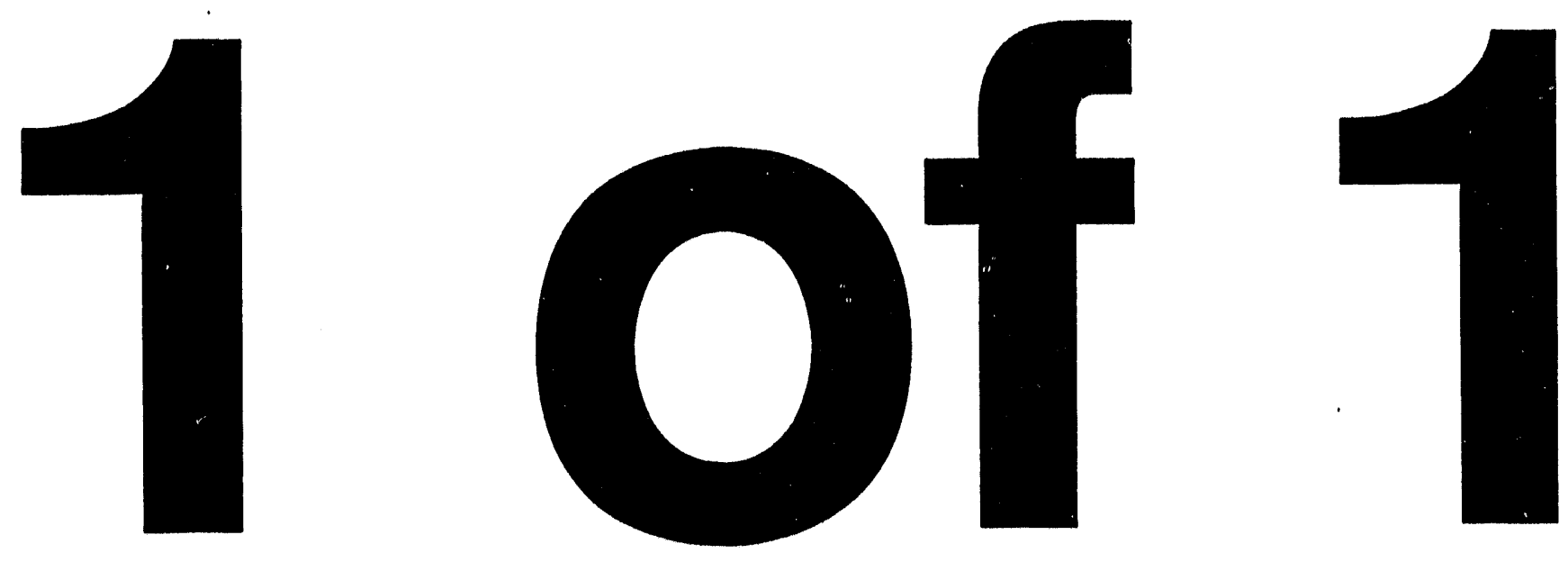
LA-UR- $\quad 93-3464$
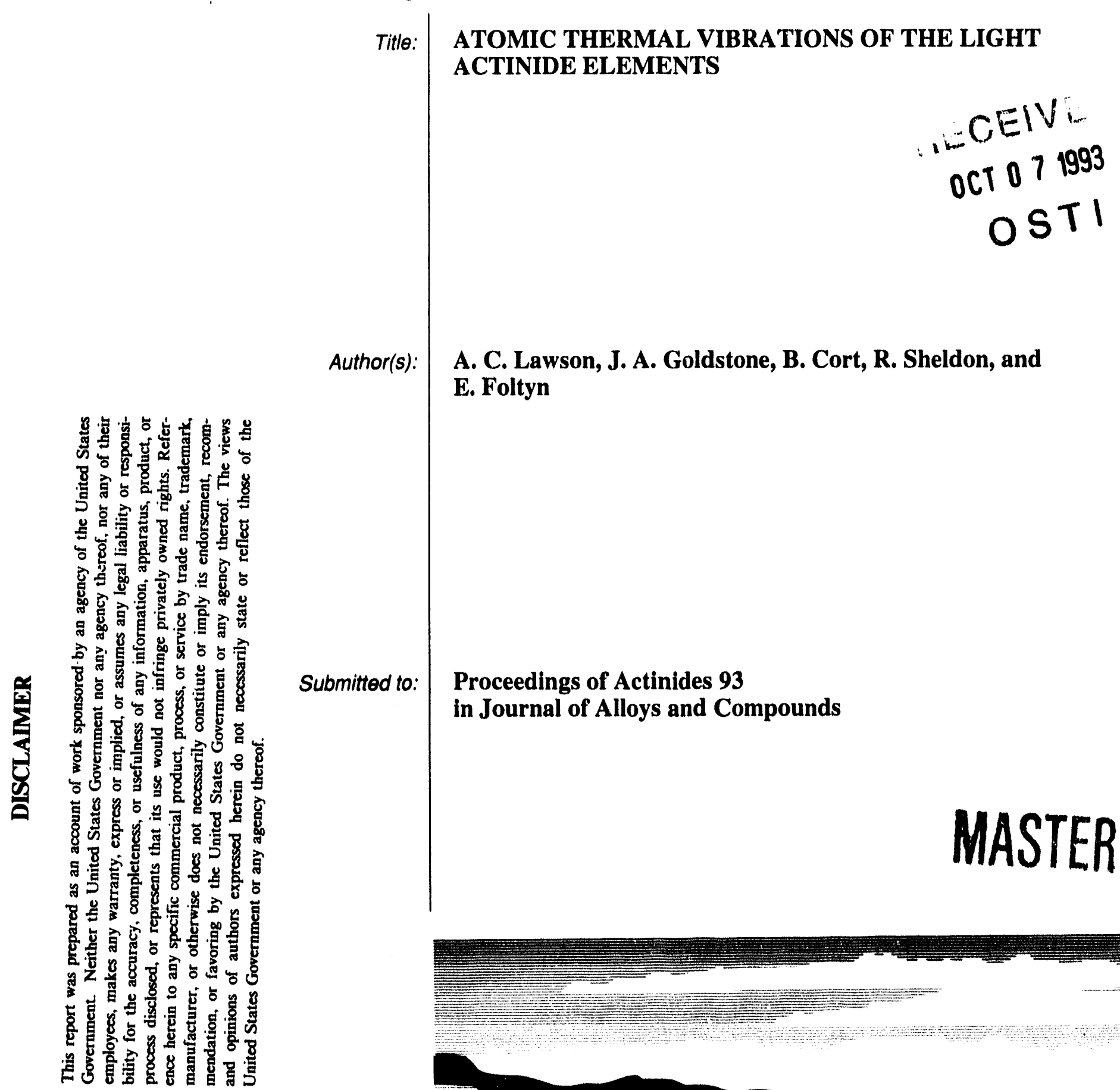

Submitted to:

Proceedings of Actinides 93

in Journal of Alloys and Compounds

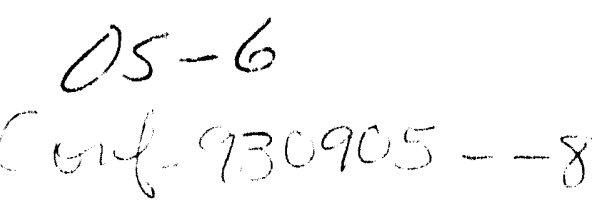

E. Foltyn

\section{MASTER}

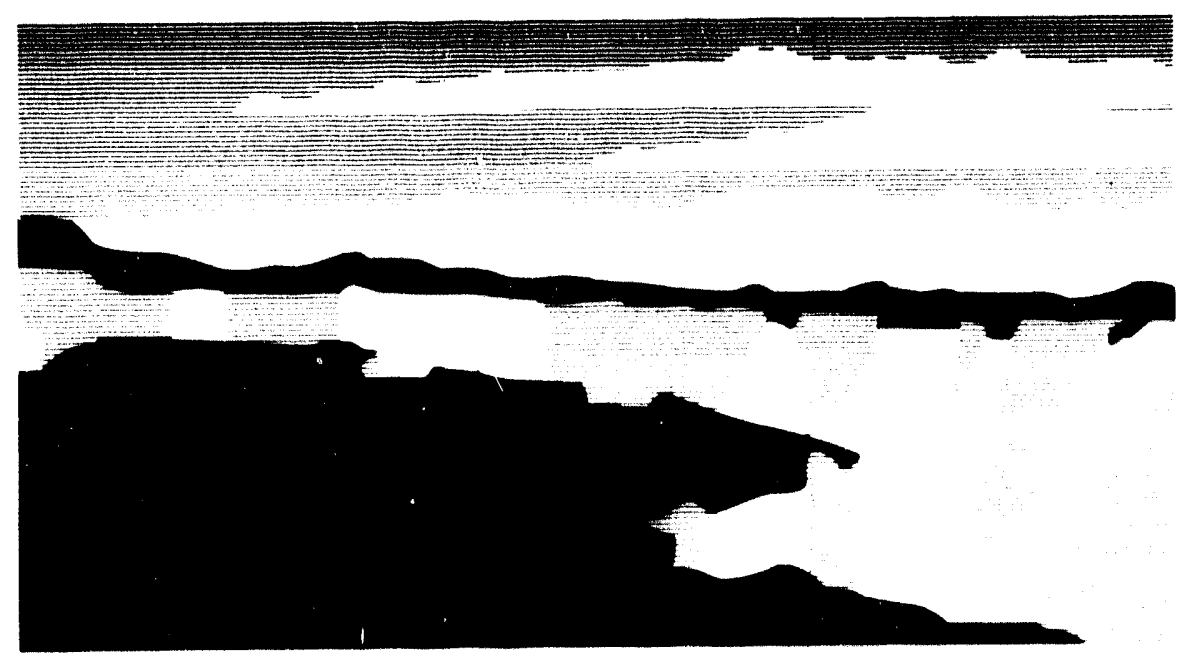

Los Alamos National Laboratory, an aftirmative action/equal opportunity emplóyer, is operated by the University of California for the U.S. Department of Energy Los Alamos National Laboratory, an aftirmative action/equal opportunity employer, is operated by the University of Calitornia tor the U.S. Department of Energy publish or reproduce the published form of this contribution, or to allow others to do so, for U.S. Government purposes. The Los Alamos National Laboratory requests that the publisher identify this article as work performed under the auspices of the U.S. Department of Energy. 


\title{
ATOMIC THERMAL VIBRATIONS OF THE LIGHT ACTINIDE ELEMENTS
}

\author{
A. C. Lawson, J. A. Goldstone, B. Cort, R. I. Sheldon, and E. M. Foltyn \\ Los Alamos National Laboratory \\ Los Alamos, New Mexico 87545
}

\section{Abstract}

We have been using time-of-flight neutron powder diffraction to determine the DebyeWaller factors of the light actinide elements. The Debye-Waller factor is a measure of the mean-square atomic displacement that arises from the thermal motion of the atoms in any solid. Its temperature dependence determines a Debye-Waller temperature, $\Theta_{D W}$, that is characteristic of the elastic properties of the solid. The data are obtained by Rietveld analysis of neutron diffraction powder patterns obtained at several temperatures. We have analyzed the results for $\alpha-U, \alpha-N p, \alpha-P u$ and $\delta-P u_{0.95} \mathrm{Al}_{0.05}$. The measured temperature dependence of the $\Theta_{D W}$ 's can be interpreted with an "effective" Grüneisen constant, whose numerical values are much too high to be explained with harmonic forces.

The primary data in a Debye-Waller experiment are neutron diffraction data that have been fitted with the Rietveld refinement technique $[1,2]$. The effect of thermal vibrations on the diffraction pattern is quite strong, with a d-spacing dependence that is given by

$$
l \propto e^{-\frac{8 \pi^{2}<u^{2}>}{d^{2}}}
$$

so that $\left\langle u^{2}\right\rangle$, the mean-square thermal displacement, is easily deterrnined by Rietveld refinement. $\left\langle u^{2}>\right.$ is related to a characteristic parameter of the solid, the Debye-Waller temperature, $\Theta_{\mathrm{DW}}$, by

$$
<u^{2}>_{\text {iddeal }}=\frac{3 \hbar^{2} T}{m k_{B} \Theta_{D W}^{2}}\left\{\frac{\Theta_{D W}}{4 T}+\frac{T}{\Theta_{D W}} \int_{0}^{\frac{\Theta_{D W}}{T}} \frac{u d u}{e^{u}-1}\right\}+<u^{2}>_{\text {offset }}
$$


where $k_{B}$ is Boltzmann's constant, $h$ is Planck's constant, and $T$ is the absolute temperature. This is the standard equation for the thermal vibrations of a monatomic cubic solid $[3,4]$; it has been modified by the addition of an extra zero-point motion, $\left\langle u^{2}\right\rangle_{\text {offset, }}$ that is required to fit the data. We believe that the offset is an artifact caused by difficulties in fitting the background neutron scattering in the diffraction patterns. More than one offset is required if data are taken under differing experimental conditions, e.g., if both a furnace and a cryostat are used. The offset drops out of the analysis when the temperature dependence of $\left\langle u^{2}\right\rangle$ is considered. Eq. (2) is too simple to be correct in principle for a real actinide structure. However, it seems to give useful results in practice.

We have found that it is necessary to allow $\Theta_{D W}$ to be slightly temperature dependent. This is necessary in principle to allow for the changes in the elastic constants that are induced by the volume changes that accompany ordinary thermal expansion, an effect described by the Grüneisen constant in the quasi-harmonic approximation of lattice dynamics. We have made the empirical assumption that

$$
\Theta_{D W}=\Theta_{D W .0}+c T
$$

Our results for $\alpha-U, \alpha-\mathrm{Np}, \alpha-\mathrm{Pu}$ and $\delta-\mathrm{Pu}_{0.95} \mathrm{Al}_{0.05}$ are shown in Fig. 1. The lines through the data are fits to Eqs. 2 and 3, and the fitted offsets have been suppressed. The fitted values are shown in Table 1. Data involving the temperature dependence of $\Theta_{D W}$, can be analyzed in terms of the Grüneisen constant, $\gamma_{G}$. In terms of the phonon frequencies, the Grüneisen constant is given by

$$
\frac{\Delta \omega}{\omega}=\gamma_{c} \frac{\Delta \mathrm{v}}{\mathrm{v}}=-\gamma_{c} \chi T \cong \frac{\Delta \Theta_{D W}}{\Theta_{D W}}
$$

where $\Delta \omega / \omega$ is the relative change in phonon frequencies, and $\Delta v / v$ is the relative volume change. Numerical values of the Grüneisen constant are usually in the range of 1-2, so there is always some temperature dependence to be expected in $\Theta_{D W}$. An additional temperature dependence in $\Theta_{D W}$ can result from the anharmonicity arising from a quadratic 
term in the interatomic potential; the functional temperature dependence of $\Theta_{D W}$ is in this case the same as in the quasi-harmonic theory, so that the two are not distinguishable experimentally by our technique. We can, however, define an "effective" Grüneisen constant, $\gamma_{\text {eff, }}$, from experimentally determined values,

$$
\gamma_{e t,}=-\frac{c}{\chi \Theta_{D W}}
$$

Values of $\chi$ and $\gamma_{\text {eff }}$ are given in Table 1. The experimental values of $\chi$ are determined by linear approximation to the atomic volumes derived frorr our diffraction data, shown in Fig. 2.

The main point of Table 1 is that all the values of $\gamma_{\text {eff }}$ are much larger than can be expected from the quasi-harmonic effect. We conclude from this that true an-harmonic effects may be important in explaining the unusual lattice behavior - both in $\Theta_{D W}$ and in $\chi$ — of the light actinide metals.

\section{Acknowledgments}

This work was supported under the auspices of the United States Department of Energy. The Manuel Lujan, Jr., Neutron Scattering Center is a national user facility funded by the United States Department of Energy, Office of Basic Energy Sciences - Materials Science, under contract No. W-7405-ENG-36. The Intense Pulsed Neutron Source is operated as a national user facility by the United States Department of Energy, Office of Basic Energy Sciences - Materials Science, under contract No. W-31-109-ENG-38. 


\section{References}

1 A. C. Lawson, A. Williams, J. A. Goldstone, D. T. Eash, R. J. Martinez, J. I. Archuleta, D. J. Martinez, B. Cort, and M. F. Stevens, J. Less-Common Metals 167 (1991) 353.

2 A. C. Lawson, G. H. Kwei, J. A. Goldstone, B. Cort, R. I. Sheldon, E. Foltyn, J. Vaninetti, D. T. Eash, R. J. Martinez and J. I. Archuleta, in J. V. Gilfrich, T. C. Huang, C. R. Hubbard, M. R. James, R. Jenkins, G. R. Lachance, D. K. Smith and P. K. Predecki (eds.), Advances in X-Ray Analysis, Vol. 36, Plenum Press, New York, 1993, p. 577.

3. B. T. M. Willis and A. W. Pryor, Thermal Vibrations in Crystallography, Cambridge University Press, London, 1975, p. 125.

4. G. Grimvall, Thermophysical Properties of Matter, North-Holland, Amsterdam, 1982, p. 102.

5. A. C. Lawson, C. E. Olsen, J. W. Richardson, Jr., M. H. Mueller and G. H. Lander, Acía Cryst. B44 (1988) 89-96.

o. A. C. Lawson, C. E. Olsen, J. A. Goldstone, J. W. Richardson, M. H. Mueller and R. L. Hitterman, High and Low Temperature Powder Diffraction Study of Alpha- Uranium, IPNS Progress Report 1986-1988, 1988, p. 37 (Argonne National Laboratory).

7. J. A. Goldstone, A. C. Lawson, B. Cort and E. Foltyn, Neutron Diffraction Study of Alpha, Beta and Gamma Phases of Neptunium, LANSCE Experiment Reports - 1990 Run Cycle, LA-12194-PR, 1991, p. 98 (Los Alamos National Laboratory). 
Lawson, Goldstone, Colt, Sheldon and Foltyn, Atomic Thermal Vibrations of the Light Actinide Elements page 5

8. J. A. Goldstone, A. C. Lawson, L. Diebolt, B. Cot and E. Foltyn, Low Temperature Study of Elemental Neptuniuri., LANSCE Experiment Reports - 1991 Run Cycle, LA-12407-PR, 1992, p 31 (Los Alamos National Laboratory).

9. A. C. Lawson, J. A. Goldstone, B. Cot and R. B. Yon Dreele, Debye-Waller Factors of $\alpha$ Plutonium, LANSCE Experiment Reports, 1992 Run Cycle, submitted for publication, (Los Alamos National Laboratory).

10. A. C. Lawson, J. Vaninetti, J. A. Goldstone, R. I. Sheldon and B. Cot, Debye-Waller Factors of $\delta$-phase $\mathrm{Pu}_{0.95} \mathrm{Al}_{0.05}$ Between 15 and $900 \mathrm{~K}$, LANSCE Experiment Reports - 1990 Run Cycle, LA-12194-PR, 1991, p 128, ( Los Alamo National Laboratory). 


\section{Table 1.}

Debye-Waller parameters and effective Grüneisen constants of the light actinide elements.

\begin{tabular}{lccccc} 
& References & $\Theta_{0 w 0}(K)$ & $c$ & $\chi\left(10^{-6} K^{-1}\right)$ & $\gamma_{\text {eff }}$ \\
\hline$\alpha-U$ & 5,6 & $260(4)$ & $-0.109(4)$ & 50.2 & 8.3 \\
$\alpha-\mathrm{Np}$ & 7,8 & $269(10)$ & $-0.15(2)$ & 59.3 & 9.4 \\
$\alpha-\mathrm{Pu}$ & 9 & $201(5)$ & $-0.08(2)$ & 96.4 & 4.1 \\
$\delta-\mathrm{Pu}$ & 10 & $132(1)$ & $-0.047(1)$ & 20.3 & 17.5
\end{tabular}




\section{Fiqure Captions}

Fig. 1. Mean-square thermal vibrations versus temperature of the light actinide elements The fit through the points is described in the text.

Fig. 2. Atomic volumes versus temperature of the light actinide elements. The dotted lines are linear approximations to the thermal expansion. 


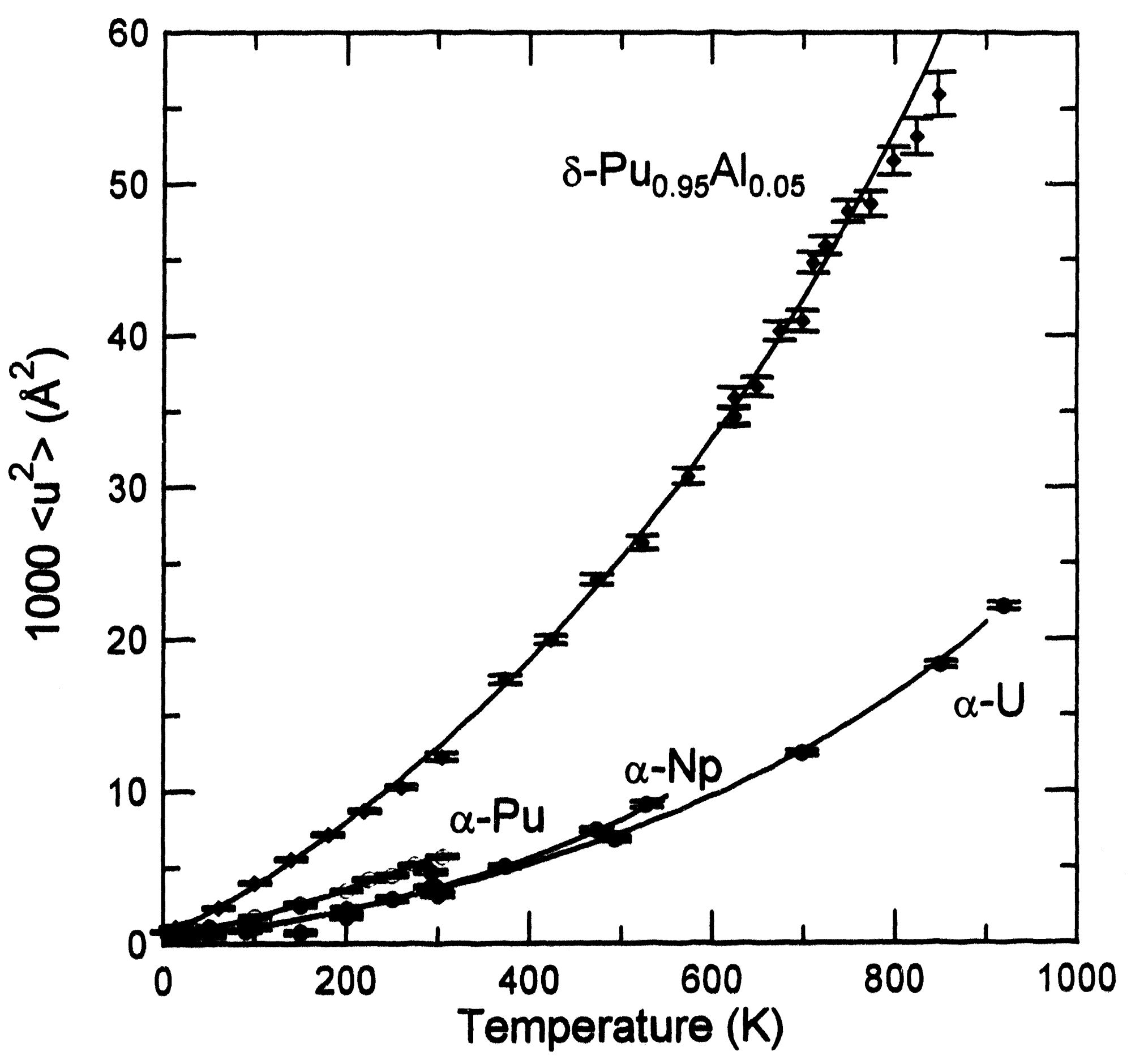




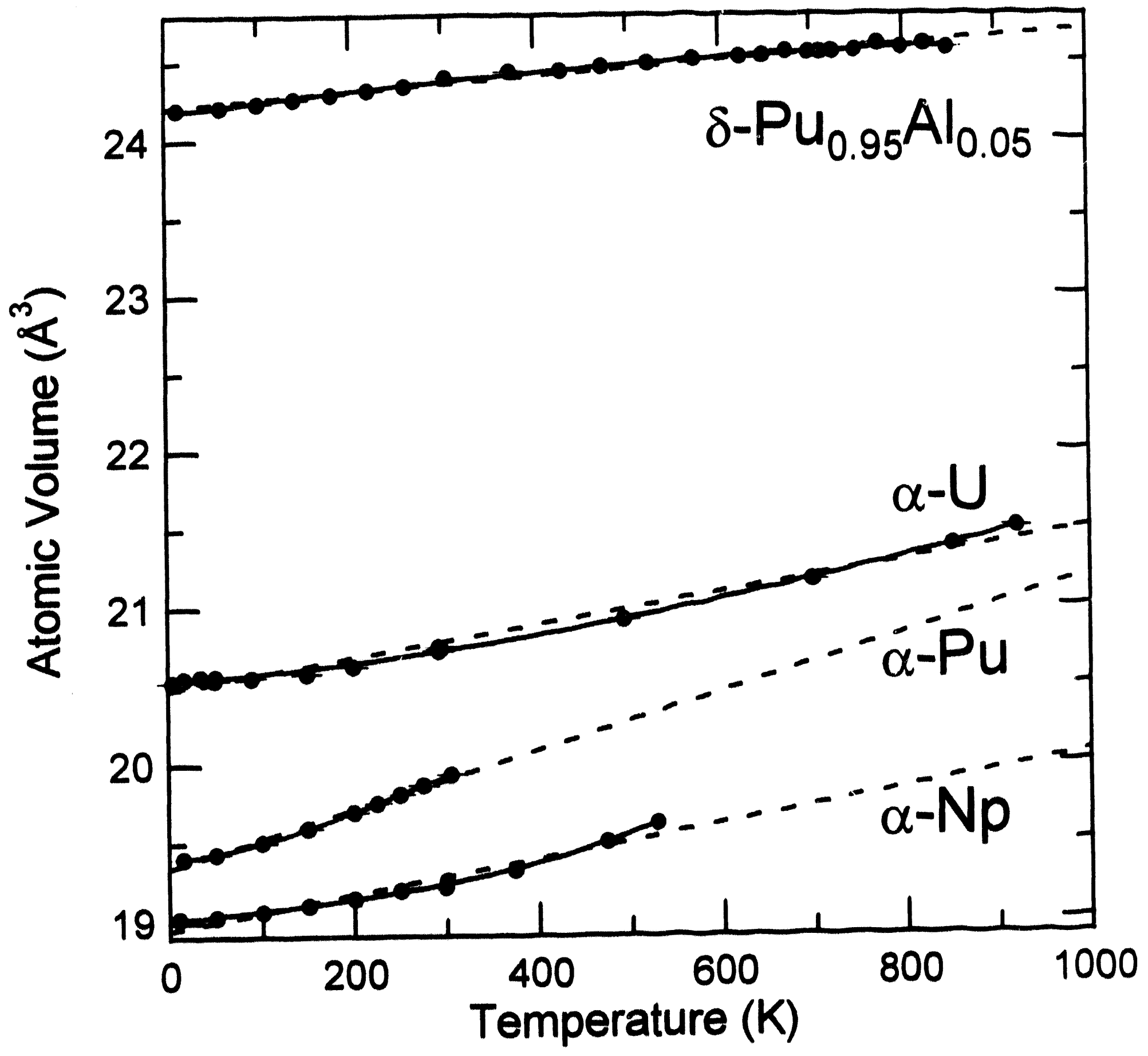


